\title{
Arsenic BCR Three-Step Sequential Extraction and Microbial Community's Response Research in Soil Polluted by Steel-Smelting with Depth
}

\author{
Li-Kun LI ${ }^{a}$, Ling-Chen KANG, Li-Jun LU, Lei ZHANG,Gai-Feng XUE \\ Environmental Protection Institute of WISCO, 430080, Wuhan, China \\ Li-Kun Li, *a670608677@qq.com
}

Keywords: Arsenic, Microbial response, Sequential extraction, Soil

\begin{abstract}
Five soil samples in different depths of a site in the close vicinity of a Wuhan iron and steel factory (Core 1) were collected. One uncontaminated soil profile (Core 2) was taken for reference purpose. The soil arsenic content and speciation vertical distribution patterns were analyzed by inductively coupled plasma-mass spectrometry, and BCR sequential extraction protocol. The results showed that the arsenic content in Core 1 was remarkably higher than Core 2 in same depth, which indicated that microbial communities were very sensitively to indicate arsenic distribution and toxicity. Additionally, principal component analyses also revealed that the arsenic exchangeable fraction play an important role to indicate arsenic ecotoxicity in mining soil and sediment.
\end{abstract}

\section{Introduction}

Environmental concern on arsenic (As) contamination has increased in the past few decades due to the increased awareness of as risk to plants, animal and human health. In most previous researches, focuses are mainly on investigating the total arsenic content in soil and sediment. This method is useful for evaluating the arsenic burden, however arsenic mobility depends strongly on the specific chemical species or bonding involved. consquently sequential extraction procedures which is a good compromise method that gives information on the environmental contamination risk[1,2].

Today, the Community Bureau of Reference (BCR) sequential extraction procedures have been widely applied to soils and aquatic sediments to characterize their chemical phase associations [3-4]. Despite experimental uncertainty with all presently available methods for solid phase metal fractionation $[5,6]$, they are probably the most useful tool for predicting longterm adverse effects from contaminated solid material [7]. Most analytical and bioanalytical chemical techniques can be explored to assess the results of sequential extraction and then to confirm the distribution patterns of metals in soil profiles [8]. These researches can help us understand the geochemical processes governing arsenic mobilization and potential ecological significance. Additionally, the feedback of soil microcosm on arsenic ecotoxicity is a very useful indicator to assess the hazard and toxicity of arsenic to our environment.

The main purpose of this research was to investigate the arsenic vertical speciation distribution in steel smelting soil profiles using a BCR three-step sequential extraction procedure and also to examine a rapid response of microbial community by colony forming unit protocol, respectively. It is important to study how arsenic speciation changes with soil depth and their influence to microbial response. In the end, by compared contaminated and uncontaminated soil profile, these analyses would be helpful to develop our cognition in arsenic dynamic and potential ecotoxicity from contaminated sludge and soil.

\section{Experimental}

\section{Materials}

Two soil profiles were collected in Wuhan in Wuhan iron and steel factory, they were immediately put into plastic bags to prevent oxidation in the material at $277 \mathrm{~K}$. All samples from one depth were 
mixed. And then, they were immediately put into plastic bags to prevent oxidation in the material at $277 \mathrm{~K}$. Afterwards, these samples were immediately transported back to the laboratory. In the laboratory, after air-dried and homogenized by sieving to less than $2 \mathrm{~mm}$ to remove roots and large objects in sterile condition, soil samples were stored at $277 \mathrm{~K}$. Each soil sample was divided into two portions for biological and physicochemical studies, respectively. The portion for biological properties determination was air-dried at $301 \mathrm{~K}$, and the portion for physicochemical properties determination was oven-dried at $373 \mathrm{~K}$.

\section{Arsenic Sequential Extraction Procedure}

First step (Exchangeable and weak acid soluble fraction): $1 \mathrm{~g}$ soil sample was extracted with $40 \mathrm{~mL}$ of acetic acid $(0.11 \mathrm{M}, \mathrm{pH}=2.82)$ solution by shaking in a mechanical, end-over-end shaker at $30 \pm$ $10 \mathrm{rpm}$ for $16 \mathrm{~h}$ at room temperature. The extract was separated from the solid residue by centrifugation at $3000 \mathrm{rpm}$ for $20 \mathrm{~min}$. The supernatant was collected into a beaker. The residue was treated similarly by adding doubly deionised water $(10 \mathrm{~mL} \times 2)$ as described above, but shaking only for 15 min. The supernatant was added to the beaker while taking special care to avoid any introduction of the solid residues.

Second step (Reducible fraction): $40 \mathrm{~mL}$ of $0.5 \mathrm{M}$ hydroxylammonium chloride solution (adjusted to $\mathrm{pH}=1.5$ with $2 \mathrm{M}$ nitric acid) was added to the residue from the first step, and the mixture was shaken $30 \pm 10 \mathrm{rpm}$ for $16 \mathrm{~h}$ at room temperature. The acidification of this reagent was completed by adding a $2.5 \%(\mathrm{v} / \mathrm{v}) 2 \mathrm{M} \mathrm{HNO}_{3}$ solutions. The extract was separated and the residue was washed as in the first step.

Third step (Oxidizable fraction): $10 \mathrm{~mL}$ of $8.8 \mathrm{M}$ hydrogen peroxide solution was carefully added into the residue from the second step. The mixture was digested for one hour at room temperature and another hour at $358 \mathrm{~K}$, and the volume was reduced to less than $2 \mathrm{~mL}$. A second aliquot of 10 $\mathrm{mL}$ of $\mathrm{H}_{2} \mathrm{O}_{2}$ was added, the mixture was digested for $1 \mathrm{~h}$ at $358 \mathrm{~K}$, and the volume was reduced to about $1 \mathrm{~mL}$. The residue was extracted with $50 \mathrm{~mL}$ of ammonium acetate $(1 \mathrm{M}$ adjusted to $\mathrm{pH} 2.0$ with conc. nitric acid) were added into the residue. The mixture was shaken on a magnetic stirrer at the speed of $30 \mathrm{rpm}$ for $16 \mathrm{~h}$ at room temperature.

Arsenic determination of extracts

$2 \mathrm{~mL}$ of $\mathrm{HNO}_{3}(65 \%)$ was added into the final extracts acquired from each step and the solutions were evaporated to almost dryness. Finally, $20 \mathrm{~mL}$ of aqua regia $\left(3: 1 \mathrm{conc}\right.$. $\mathrm{HCl} / \mathrm{conc}$. $\left.\mathrm{HNO}_{3}\right)$ were added to the extracted and followed by microwave-assisted digestion. The arsenic content of the digested extracts were determined by the quadrupole ICP-MS system. Control samples were also processed and they were treated in exactly the same way as the test samples. In each experiment, four replicate test samples and two control samples were processed simultaneously. Blank tubes (containing reagent but no soils) were also taken through each complete BCR procedure.

\section{Soil Microbial Communities}

The number of living microorganisms of mixed population was estimated by viable count on serial spread plates. Briefly, $0.1 \mathrm{~mL}$ of each serial dilution of sample suspension was spread over an agar ( $2 \%)$ plate with beef extract peptone medium $(0.05 \%$ beef extract, $0.05 \%$ peptone, $0.03 \% \mathrm{NaCl}$, w/v) containing $0.05 \%$ fungicidine for culturing bacteria, Martin’s medium ( $1 \%$ glucose, $0.5 \%$ peptone, $0.1 \% \mathrm{~K} 2 \mathrm{HPO} 4,0.05 \% \mathrm{MgSO} 4 \cdot 7 \mathrm{H} 2 \mathrm{O}$, and $0.003 \% \mathrm{w} / \mathrm{v}$ Rose Bengal) containing $0.003 \%$ of streptomycin for culturing fungus (Aon M A et al., 2001) and Gause's No. 1 synthetic medium (0.1 \% KNO3, 2 \% soluble starch, $0.05 \%$ K2HPO4, $0.05 \% \mathrm{MgSO} 4 \cdot 7 \mathrm{H} 2 \mathrm{O}, 0.05 \% \mathrm{NaCl}$, $0.001 \% \mathrm{w} / \mathrm{v}$ FeSO4) for culturing actinomycetes(Zheng S X et al., 2007 ). Six continuous 10-fold dilutions of each sample were prepared starting with $9.0 \mathrm{~mL}$ sterilized phosphate buffered saline and $1.0 \mathrm{~g}$ of soil sample. The soil samples were taken in a sterile flask and all planting process were aseptically performed. After inoculation, plates (duplicates) were incubated either aerobically (total bacteria, fungi, and actinomycetes) or under microaerophilic conditions at $301 \mathrm{~K}$. Microaerophilic incubation was carried out in an anaerobic glovebox (Coy Laboratories Products, Toepffer GmbH, Goppingen, Germany). Latter procedures were triplicate. All plates were incubated at $301 \mathrm{~K}$ in the 
dark until colonies appeared (3 days for bacteria and 5 days for fungi and actinomycetes). The CFUs varied from 10 to 100 for fungi and from 30 to 300 for prokaryotes.

\section{Characterization}

All data were done in triplicate and mean values were taken for statistical analysis. The results obtained from various methods were analyzed by one-factor ANOVA and followed by the t-test using the Excel data analyses tool (Microsoft, Redmond, WA, USA). The statistical significance level was set at $\mathrm{P}<0.05$. Principal component analyses (PCA) and correlation analyses (CA) were performed using the software version 12.0 SPSS for Windows (Chicago, IL, USA) and Origin 7.5 (OriginLab, MA, USA), respectively. A correlation was deemed to be significant when $r>0.5$. PCA is the simplest of the true eigenvector-based multivariate analyses and can been considered as revealing an internal structure of the data in a way that can well explain the variance in the data.

\section{Results and Discussion}

\section{Sequential Extraction Results}

In this study, arsenic fractionation pattern and vertical speciation distribution were analyzed by BCR three-step sequential extraction procedure and depicted in Table 1.

Table 1 Arsenic extraction yields (\%) of BCR three-step sequential extraction

\begin{tabular}{|c|c|c|c|c|}
\hline \multirow{2}{*}{$\begin{array}{l}\text { Sampling } \\
\text { depth (cm) }\end{array}$} & \multicolumn{4}{|l|}{ Core 1} \\
\hline & Exchangeable (\%) & Reducible (\%) & Oxidisable (\%) & Residual (\%) \\
\hline $0-20$ & 17 & 21 & 30 & 32 \\
\hline $20-40$ & 14 & 30 & 20 & 36 \\
\hline $40-60$ & 10 & 29 & 22 & 39 \\
\hline $60-80$ & 8 & 28 & 22 & 42 \\
\hline $80-100$ & 6 & 26 & 24 & 44 \\
\hline \multirow{2}{*}{$\begin{array}{l}\text { Sampling } \\
\text { depth (cm) }\end{array}$} & \multicolumn{4}{|l|}{ Core 2} \\
\hline & Exchangeable (\%) & Reducible (\%) & Oxidisable (\%) & Residual (\%) \\
\hline $0-20$ & 20 & 25 & 25 & 30 \\
\hline $20-40$ & 18 & 26 & 21 & 35 \\
\hline $40-60$ & 17 & 27 & 19 & 37 \\
\hline $60-80$ & 15 & 24 & 20 & 41 \\
\hline $80-100$ & 10 & 25 & 22 & 43 \\
\hline
\end{tabular}

The soil as content was divided into four different fractions after the BCR three-step sequential extraction procedure. Száková (1999) reported that $4 \%$ arsenic was extractable in the first step of the procedure, $9 \%$ and $12 \%$ arsenic were extracted in the second and third steps, respectively, and $75 \%$ arsenic remained in the residual fraction. Similarly, all four different fractions of arsenic in the soil samples were analyzed in this research and summarized in Table 1 . It was found that the arsenic content in the exchangeable fraction was highest at the soil surface layer and it was then gradually decreased with depth. In our results, the exchangeable fraction was $17 \%$ and $20 \%$ in Cores 1 and 2 surface layer, respectively. This result was significantly higher than Száková's work. Our results indicate that As is easily migrated at the top soil surface layer which can be explained by two main reasons. The first reason was due to the lower $\mathrm{pH}$ at the top soil layer. It is well-known that As or other metals are presented in minerals as molecule form. In these minerals, anions (such as $\mathrm{S}^{2-}$, $\mathrm{Cl}^{-}, \mathrm{SO}_{4}{ }^{2-}, \mathrm{CO}_{3}{ }^{2-}, \mathrm{SiO}_{4}{ }^{4-}$, and $\mathrm{Si}_{2} \mathrm{O}_{7}{ }^{6-}$ ) can bond with some cations with large ionic radii (such as $\mathrm{As}^{3+}, \mathrm{As}^{5+}, \mathrm{Pb}^{2+}$, and $\mathrm{Cd}^{2+}$ ) by various chemical bonds such as covalent, coordinate, and ionic bonds. These chemical bonds are commonly very stable; however, they are also easy to break up at lower $\mathrm{pH}$. The soil at the surface as shown in Table 1 is more acidic, inferring that As or other metals will be loosely bounded and unstable at the surface layer. Secondly, some organic matter influence also 
needed to be investigated. To this part, our results showed that more organic matter has been measured at the top soil layer (Table 1). Moreover, the organic matter was radually decreased with depth. The vertical distribution of organic matter also was very similar to arsenic content in Cores 1 and 2. This phenomenon indicated that organic matter has a strong potential to mobilize arsenic from soils and sediments.

The as contents in the reducible and oxidizable fractions of soils at different depths slightly varies in both locations Cores 1 and 2. The as content does not change much with the soil depth. These results may be related to human activities, soil biological activities or geological processes such as lithological properties or groundwater effect. However, the As content in every single soil layers shows the order: residual > reducible $>$ oxidizable $>$ exchangeable. Higher As contents were found in the residual fraction of the subsoil samples than their corresponding surface layers and the As concentration increases with the depth of the soil. This observation is reasonable since weathering processes are generally more intense in soil surface than the deeper ones. Secondly, As will be easier to leach out as the soil $\mathrm{pH}$ is lower at the top soil surface layer. The As profile at the two locations Cores 1 and 2 are very similar probably because they are not very far apart $(8 \mathrm{~km})$ so that the weathering processes are quite similar at these low locations.

\section{Multi-Parameter Analyses}

PCA is a multivariate method that reduces the dimensionality of a data set while maintaining data variability information. In this study, CA and PCA methods were applied to reduce and correlate the as sequential extraction parameters and then more useful data and helpful information can be extracted. Figure 1 shows the PCA of As BCR sequential extraction and soil microorganisms the parameters at Cores 1 and 2.
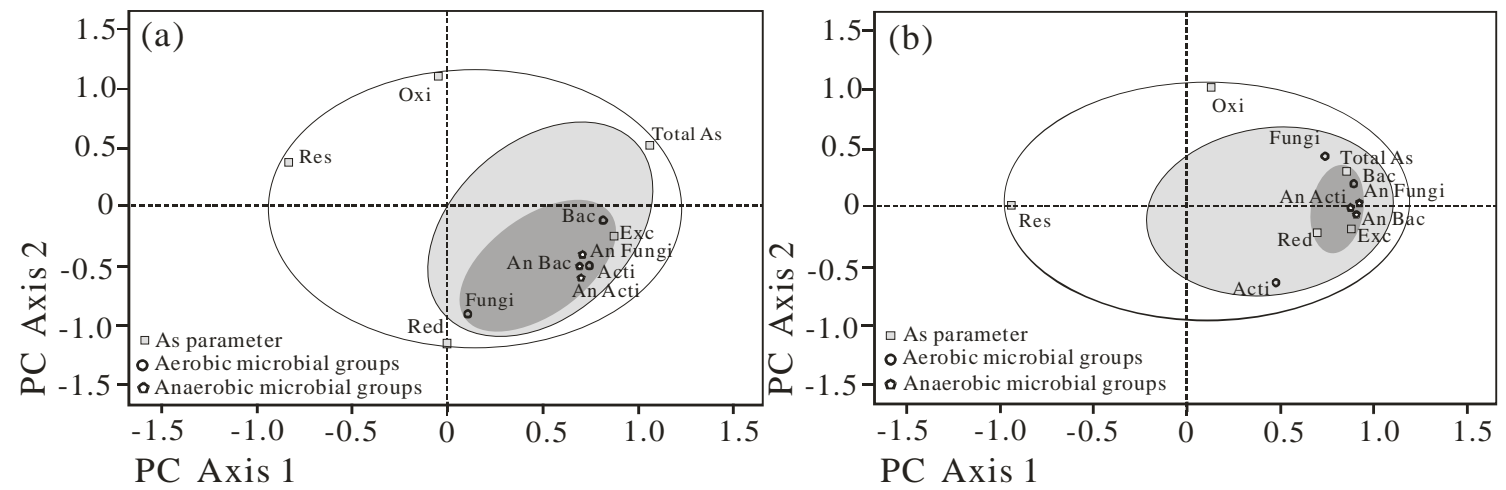

Figure 1 Principal component analysis of multi-parameter at Cores 1 and 2. The arsenic content and microbial response in the (a) at Core 1. The arsenic content and microbial response in the (b) at Core 2. The abbreviations for multi-parameters and (full name) are as follows: As parameter contained Exc (Exchangeable fraction), Red (Reducible fraction), Oxi (Oxidizable fraction), Res (Residual fraction), and Total As (Arsenic total content). Aerobic microbial groups contained Bac (Bacteria) and Acti (actinomycetes). Anaerobic microbial groups contained An Bac (Anaerobic Bacteria), An fungi (Anaerobic fungi) and An Acti (Anaerobic actinomycetes).

The PCA result show some similarities and differences between the multi-parameter. The largest differences are scope of reference ring and intensity of multi-parameter. At Cores 1 and 2, the largest ring is mainly determined by the As oxidizable and residual fractions. This result clearly demonstrates that the As oxidizable and residual fractions are not significantly correlated with the microbial response. In order to estimate the relevance of other factors, a medium ring was performed with the same area as shown in Figure 1. In the Core 1, the parameters of total As content and As reducible fraction had been already eliminated from the medium ring; however, they existed in the medium ring at Core 2. This result illustrates that human activity is the dominant factor affecting the As distribution and enrichment in short term. Moreover, soil microorganism also plays a key role in As migration, especially in an uncontaminated soil or in a long term 
situation. In the end, the minimal cycle was preformed to investigate the main component of multi-microbial response. By contrast, the size of minimal cycle at Core 1 is significantly larger than at Core 2, indicating that the soil microbial groups have taken place a great change due to the anthropogenic factors. Furthermore, it shows that more metabolic diversity of microbial groups has been found in aerobic and anaerobic processes. This result was similar to Singer's work, who pointed out that soil microflora had been interfered during human activity. Since As exchangeable fraction also had been included in minimal cycle, this result clearly indicates that the As exchangeable fraction is significantly correlated with microbial response. Thus, these results demonstrate that the As exchangeable fraction is the best parameter to reveal As ecotoxicity in soil or sediment.

\section{Conclusion}

In summary, a sensitive method for the detection of As enrichment and ecotoxicity with depth profile has been developed on the basis of BCR sequential extraction and soil microbial communities using soil samples from the contaminated and unpolluted locations. The microbial responses were assessed by plate counting methods. The aim of this study was to demonstrate that the system developed was versatile and can be employed for continuous measurement and assessed the extent of As pollution. The optimization of the system for other metal ecotoxicity and microbial sensitive responses will be conducted in the near future in our laboratories.

\section{Acknowledgement}

This work was supported by the Fundamental Research Funds for the Wuhan Iron and Steel (Group) Company.

\section{References}

[1] A. Tessier , Pg. G. C. Campbell, M. Bisson, Sequential extraction procedure for the speciation of particulate trace metals, Anal. Chem. 51 (1979) 844-851.

[2] C. Gleyzes, S. Tellier, M. Astruc, Fractionation studies of trace elements in contaminated soils and sediments: a review of sequential extraction procedures, TRAC-Trend Anal. Chem. 21(2002) 451-467.

[3] V. H. Kennedy, A. L. Sanchez, D. H. Oughton, A. P. Rowland, Use of single and sequential chemical extraction to assess radionuclide and heavy metal availability from soils for root uptake, Analyst 122(1997) 89-100.

[4] M. Pueyo, J. Mateu, A. Rigol, M. Vidal, J. F. Loópez-Sánchez, G. Rauret, Use of the modified BCR three- step sequential extraction procedure for the study of trace element dynamics in contaminated soils, Environ. Pollution 152(2008)330-341.

[5] M. D. Ho, G. J. Evans, Sequential extraction of metal contaminated soils with radiochemical assessment of readsorption effects, Environ. Sci. \& Technol. 34(2000)1030-1035.

[6] C. Whalley, A. Grant, Assessment of phase selectivity of the european community bureau of reference (bcr) sequential extraction procedure for metals in sediment, Anal. Chimi. Acta 291(1994)287-295.

[7] C. Kheboian, C. F. Bauer, Accuracy of selective extraction procedures for metal speciation in model aquatic sedimentsc, Anal. Chem. 59(1987)1417-1423.

[8] M. Raksasataya, A.G. Langdon, N. D.Kim, Assessment of the extent of lead redistribution during sequential extraction by two different methods, Anal. Chimi. Acta 332(1996)1-14. 